taining eight double benches (I I feet by 5 feet), so that sixty-four students can work simultaneously; adjoining are balance rooms and a combustion room. The laboratories are well provided with fume cupboards, placed in the window recesses, and ventilated by two main electric "blowers " placed in dormers at the ends of the building. On the same floor is the departmental library ( 26 feet by 5 feet), which contains complete sets of all the more important English and foreign chemical periodicals, in addition to important general works of reference. The main lecture theatre is situated at the back of the building on the first floor; it is provided with lift-up seats for I30 students, and adjoining it are the preparation room and museum.

On the ground floor are the following rooms:- the physical chemical laboratory, the director's private room and private laboratory, distillation room, two dark rooms, general stores, special stores, porter's room, demonstrator's private laboratory, lecture room with accommodation for fifty students, and two small research rooms. In the basement are a small metallurgical laboratory, extra stores, a fire-proof room, a small dynamo room, and a mechanic's room.

The physical chemical laboratory has a central table 12 teet by 5 feet, with no reagent shelves. Around the walls are slate slabs and wooden tables. The slate tables

when the main gas supply is shut off for the night. The general heating is by means of hot-water pipes, and the lighting by means of tantalum lamps.

The laboratories are especially arranged to give students a sound training in the various branches of chemical study and are also admirably suited for the carrying out of original investigations. At present about eighty students are working in the laboratories, and of these five are engaged in research work, mainly on the relationship between constitution and the velocity of reaction of carboxylic acids.

\title{
SCIENTIFIC WORK OF THE LOCAL GOVERNMENT BOARD.
}

THE supplement to the thirty-fifth annual report of the Local Government Board, $1905^{-6} 6$, contains the report of the medical officer for 1905-6. The contents of this valuable volume are briefly summarised in the excellent introduction contributed by the principal medical officer, Mr. Power.

An account of the general administrative business of the medical department is given in Appendix $A$, which includes reports on the outbreaks of enteric fever a Basingstoke and at Lincoln.

Appendix B contains an account of the auxiliary scientific investigations carried out for the Board, of which three are contributed by Dr. Klein, and deal with plague. The first and second of these form a further contribution on the value of a new plague prophylactic prepared from the dried organs of plague-infected animals, as previously detailed by the author, who concludes that it would appear that the injection of rats with efficien material-the raw or the heated filtrate of emulsions of dried plague organsin appropriate doses has proved protective in as short a period as seven days against subsequent cutaneous inoculation of virulent $B$. pestis, that is, against plague infection administered in the most effective manner. Further, it appears that the protection thus afforded, though inducible so speedily, may be trusted to persist many days, and even weeks. The prophylactic having been proved to protect rats was also tested on monkeys in order to ascertain whether or not there was promise of its application to the human subject proving salutary and justifiable, and 25 milligrams of dry

are provided with several thermostats regulated for different temperatures. The room also contains a fume cupboard for electrolytic work, and a main accumulator board. This board carries the terminals of twenty Tudor cells placed in the adjacent room. The cells are charged from a smail motor generator in the basement, and are in groups of one, two, and four. The accumulator board also carries the terminals of eight working positions, four in the physical chemical laboratory and four in one of the large laboratories, and, in addition, three main terminals for the lecture theatre and the motor generator terminals. The terminals are so arranged that any position in the theatre, large laboratory, or physical chemical laboratory can be connected to any group or combination of groups of cells, and also, if necessary, to the motor generator terminals.

The distillation room has no gas connections, but has a long slate slab provided with water, steam, current $(220$ volts), and waste. It is used for the distillation of large quantities of inflammable liquids, and the source of heat is either steam or electric current. The fire-proof room is furnished with slate slabs and an iron fume cupboard. Experiments necessitating the use of gas during the night are conducted in this room. The gas connections for this room and for the chemical physical laboratory are so arranged that flames may be left burning in these rooms

NO. I 993, vor. 77 ? material sufficed to protect against a supra-lethal dose of virulent plague bacilli. As regards the rat, Dr. Klein finds that various species of rat are differently susceptible to the $B$. pestis, the Norway rat apparently being less susceptible than certain other races.

Dr. Gordon reports on the micrococcus of epidemic cerebro-spinal meningitis (" spotted fever"), with special reference to its identification in the upper respiratory passages. The morphological and other characters of the meningococcus are fully described, and its differentiation from other somewhat similar cocci by means of fermentation reactions on various sugars is detailed.

Dr. Sidney Martin has continued his studies on the chemical products of micro-organisms, and reports on the products of the Bacillus enteritidis sporogenes. The experiments show that the poisonous products of this organism do not consist of an endo-toxin, but of a soluble chemical and non-protein substance which is formed by the bacillus by its action on proteins. Dr. Wade contributes an exhaustive experimental inquiry on sulphur dioxide as applied in the destruction of rats and in disinfection on shipboard. The conclusion is formulated that a modification of the Clayton apparatus (described in the report) to supply dilute sulphur dioxide will prove the best adapted to practical requirements.

The last paper, by Drs. Andrewes and Gordon, discusses 
the biological characters of the staphylococci pathogenic for man, and tests that will be useful for their differentiation are described.

It will thus be seen that the report contains matter of considerable scientific and practical interest, and it would be a great pity if this work were to be discontinued in the future, as has been rumoured it may be.

\section{R. T. HEWLETT.}

\section{$M E N D E L I S M A N D S E X .^{1}$}

A LL science is founded on observed facts. All authenticated facts, no matter how observed, are valuable to science. Many invaluable facts cannot be observed without the aid of some special method, for example, experiment; but, of the total mass of facts garnered by science, data furnished by experiment form a very small part. Therefore to rely solely on experiment is to put on blinkers.

Since species are able to exist in their environments, they are adaptational forms. The more minute our knowledge of a species, the more certainly are we able to assign past or present utility to nearly all its structures and faculties. Adaptation extends deeper than structures and functions. Variability itself is adaptive. A greater or lesser degree of variability is a variation and material for natural selection. There is satisfactory evidence that the average degree of variability displayed by every species and structure is controlled by selection. The mass of variations are "spontaneous." Thus there are hundreds of human races and diseases, and every race is resistant to every lethal disease in proportion to its past experience of it. Therefore in this case there is adaptation. Therefore it is clear that the poisons of disease, no matter how virulent or universally experienced by the race, do not cause alteration in the germ-plasm, and consequent racial degeneration. On the contrary, since adaptation has occurred, it is plain that variations are spontaneous, and, since diseases are so many, that they occur all round the specific mean. But some races (e.g. European dogs in India) have been known to degenerate when removed to new environments, where the native races flourish. Therefore the insusceptibility of the germ-plasm to the direct action of the environment has been established by natural selection, and this, combined with the facts that (I) species tend to become more variable a few generations after removal from ancestral environments to which they have become closely adapted, and where, therefore, nature limits variability; (2) the degree of variability in functionally correlated structures (e.g. pairs of limbs) tends to be correlated; and (3) the greater the need for adaptation the less is the degree of variability when once adaptation has been attained (e.g. head and fore-foot of squirrels as compared to tail), affords plain evidence that variability is under the control of natural selection.

When cessation of selection as regards any character occurs, that character tends to retrogress. Therefore retrogressive variations tend to predominate over progressive variations, whereby, without an increase of mortality, species are rid of redundancies, both useless variations and old-established parts which have become useless. Since this tendency to retrogression is highly adaptive, the presumption is that it is an adaptation. 'That the retrogression which follows panmixia is not due to reversed selection is shown by the fact that, though variations favourable against all diseases occur in every human race, yet they retrogress unless preserved by selection, for races become resistant only to those diseases to which they are exposed.

The two central doctrines of Mendelism are :-(1) segregation of units, and (2) independent inheritance of characters. Taken by itself, the doctrine of segregation assigns no function to conjugation. It merely controverts the doctrine of blending. Taken with the doctrine of independent inheritance, it assigns to conjugation the function of effecting an exchange of germinal units between the two sets of parental units. That much Mendelism implies-that much and no more. Mendelians believe,

1 Abstract of a naner read before the Linnean Society on December rg, 1907, by G. Arcbdall Reid. apparently, that they have found the key to all the problems of heredity; but obviously Mendelism is concerned with nothing more than the function of conjugation. No other problem of biology with which it is concerned can be thought of. However grandiose the language used by its adherents, they are quite unable, when challenged, to indicate any other.

Mendelian inheritance is common when varieties which have arisen under artificial selection are crossed. It is comparatively rare when natural varieties (e.g. human) are crossed. Blending is then the rule. Latent traits, also, are commonly revealed by the crossing of artificial varieties. In the whole range of biological literature, no instance is recorded of a latent trait being revealed by the crossing of natural varieties. Even when artificial varieties are crossed, they never revert beyond the wild variety; that is, they never reveal traits that were latent in the wild variety. Presumably, therefore, characters become latent only under artificial selection, and consequently Mendelism is concerned, not with the main problem of conjugation, but only with certain anomalies which occur under conditions of artificial selection.

It is admitted on all hands that artificial selection is founded mainly on mutations, and that the inheritance of mutations tends to be alternative. It is admitted that the inheritance of fluctuations tends to be blended, and the evidence is conclusive that natural selection builds on fluctuations. Thus varieties are most numerous when mating individuals (e.g. birds) are enabled by good powers of locomotion to interbreed over a wide area. No interpretation of these facts save that of blending can be thought of. Human varieties, for example, arise only under conditions of geographical isolation. It has been said, on the evidence of half-a-dozen generations, that mutations are stable, and having arisen can be eliminated only by selection. This implies that only progressive variations occur in nature, and therefore that no structures ever disappear or retrogress except through reversed selection; but though variations favourable against all diseases occur in all human races, only those which are selected are preserved and contribute to evolution. Therefore it is clear that the rest retrogress, though there can be no reversed selection in this case.

When species are sexually dimorphic, mating individuals differ, as a rule, little in non-sexual characters, but much in sexual characters. Offspring reproduce either the paternal or the maternal sexual characters. That is, the reproduction of sexual characters is alternative, the male and female characters being "allelomorphic" to one another. But the inheritance of them is not alternative, for each sex inherits the characters of the other in a latent state, as is proved by a mass of evidence. Therefore, though in bi-parental reproduction there is apparently no blending as regards the sexual traits, yet the fact is that the patent characters of the one sex blend with the latent characters of the other. Sometimes the male characters are latent for a long series of generations, as in aphides, or apparently permanently, as in Cypris reptans. A mutation, like a sexual difference, is a large difference, and when an individual mutates and mates with the parent type, the reproduction of the mutation tends to be type, the resive that the inheritance is not alternative, but, on the contrary, that the ance is not alternat in those lines of descent which follow the parent type, whereas the ancestral trait is latent in those lines which follow the type of the mutant. Like the sexual traits in bi-parental reproduction, the recessive is temporarily latent in the impure dominant. Like the male characters in aphides and Cypris reptans, it is more or less permanently latent in "pure" dominants, as is the dominant character in the recessive. This is proved by the occasional occurrence of recessives in lines of "pure" dominants, and vice versa. It is even more decisively proved by the reproduction of latent ancestral characters, especially when (artificial) varieties are crossed. Cuénot's theory of colour factors attempts to interpret in Mendelian terms this fact of the reproduction of latent ancestral traits, but his hynothesis totally fails to account . for the reappearance of latent ancestral traits in pure lines of descent, as, for example, when an aged female bantam reproduces, not the secondary male characters of her own variety, but those of an ancestral type. Here there can 